\title{
Analysis of shear flexible layered isotropic and composite shells by 'EPSA'
}

\author{
Pawel Woelke ${ }^{\mathrm{a}, *}$, Ka-Kin Chan ${ }^{\mathrm{a}}$, Raymond Daddazio ${ }^{\mathrm{a}}$, Najib Abboud ${ }^{\mathrm{a}}$ and George Z. Voyiadjis ${ }^{\mathrm{b}}$ \\ ${ }^{a}$ Weidlinger Associates, Inc., New York, NY, USA \\ ${ }^{\mathrm{b}}$ Department of Civil and Environmental Engineering, Louisiana State University, Baton Rouge, LA, USA
}

Received 24 April 2010

Revised 8 March 2011

\begin{abstract}
We present a simple and efficient method for the analysis of shear flexible isotropic and orthotropic composite shells. Classical thin shell constitutive equations used in the explicit finite element code EPSA to model homogenous isotropic shells using "through-the-thickness-integration" and layered orthotropic composite shells [1-3,5] are modified to account for transverse shear deformation. This effect is important in the analysis of thick plates and shells as well as composite laminates, where interlaminar effects matter. Transverse shear stresses are calculated using a linear normal strain distribution, where first the shear forces are calculated and then the stresses are calculated by means of the generalized section properties, i.e., first and second moments of area. The formulation is a generalization of the analytical method of analyzing composite beams. It is simple and computationally inexpensive, and it yields accurate results without employing higher order displacement interpolations. In the case of isotropic shells, the transverse shear stresses are distributed parabolically, based on the assumption of linear normal strain distribution through the thickness and on application of the quadratic shape function to transverse shear strains. The transverse shear stresses are included in the elastic-perfectly plastic yield function of the Huber-Mises-Hencky type.
\end{abstract}

Keywords: Thick layered plates and shells, thick composite shells, transverse shear deformation

\section{Introduction}

Our objective is to include the transverse shear strains and stresses in the analysis of isotropic shells using "through-the-thickness-integration," as well as of composite laminates, using an explicit code EPSA [1-3,5]. Only matters directly related to this objective are discussed in detail.

Shells in which the ratio of the thickness to the radius of curvature is equal or less than $1 / 50$ are usually considered to be thin. In the case of thin shells, transverse shear strains are negligible. This is true for most of the boundary conditions. Some types of loading conditions, however, cause significant shear forces regardless of the thickness of the structure. In the analysis of structures with thickness that is large compared to the other two dimensions, transverse shear stresses become significant and need to be taken into account. In this paper, elasto-plastic analysis of isotropic shells is carried out by means of a layered method, often referred to as "through-the-thickness-integration". The elastic-perfectly plastic material model is used with a Von Mises-type yield function, with accounting for transverse shear stresses.

Transverse shear strains and stresses are especially important in the analysis of composite laminates. This is because advanced filamentary composite materials are susceptible to thickness effects, as their effective transverse shear modulus is substantially smaller than the effective elastic modulus in the fiber direction. Moreover, increasing

\footnotetext{
*Corresponding author. Tel.: +1 212367 2983; Fax: +1 212497 2483; E-mail: woelke@wai.com.

${ }^{1}$ This work was funded through Weidlinger Associates internal research and development fund.
} 
use of composite laminates in various branches of the industry requires analysis of these structures beyond elastic behavior, up to failure. Composite laminates have various modes of failure: delamination, debonding, fiber cracking and matrix yielding and cracking.

One of the most common failure modes is delamination, which is driven by the transverse shear stresses. To accurately model composite plates and shells and address the issues related to failure, it is necessary to include transverse shear stresses in analyses. We use a description of shear effects based on the Mindlin-Reissner theory of plates, which has been modified to improve the accuracy of the model. The Mindlin-Reissner theory leads to a constant distribution of the transverse shear stresses across the laminate thickness. This representation is inexact, as the transverse shear stress function is actually parabolic through the thickness of the lamina and not continuous through the laminate. To improve the accuracy of shear stress prediction, the shear correction function is applied, obtaining a parabolic distribution of shear stresses across the thickness of the laminate without changing the definitions of the shell kinematics. The correction function satisfies the boundary conditions, enforcing zero shear stresses on the outer surfaces of the laminas. Although using a second-order strain function through the thickness of the composite laminate is a significant improvement over a constant one, it still results in an undesired $\mathrm{C}^{1}$ continuity through the thickness. Substantial stiffness variations of the individual laminas lead to "jumps" of the shear stress gradients at lamina interfaces. As failure analysis focuses on stresses, we adopt a transformed section method that assumes a continuous shear strain distribution. This allows prediction of the aforementioned "jumps" in the shear stress gradients through the thickness of the composite laminate.

This paper is organized into six sections. Following the Introduction, Section 2 discusses shell kinematics. Section 3 presents the constitutive equations. Section 4 discusses yield surface for layered isotropic shells. Section 5 presents numerical examples, demonstrating that the current computational model provides accurate results. Finally, Section 6 contains the summary and conclusion.

\section{Kinematics of the shell}

The thick shell formulation is, according to Reddy's classification [24-27], a single-layer shear deformation one. It accounts for a constant state of transverse shear strains across the thickness of a composite laminate. It is not a layerwise formulation, which requires adoption of higher order interpolation functions to approximate variation of axial displacement through the thickness of the composite laminate. The use of a layerwise formulation to model composite laminates in EPSA would require major changes in the kinematics of the shell element, which could negatively affect code reliability.

The displacement-based shear deformation formulations are developed using displacement field of the form:

$$
\begin{gathered}
u(x, y, z, t)=u_{0}-z\left[-\alpha \frac{\partial w}{\partial x}-\beta \phi_{x}-\gamma \frac{4}{3}\left(\frac{z}{h}\right)^{2}\left(\phi_{x}+\frac{\partial w}{\partial x}\right)\right] \\
v(x, y, z, t)=v_{0}-z\left[-\alpha \frac{\partial w}{\partial y}+\beta \phi_{y}-\gamma \frac{4}{3}\left(\frac{z}{h}\right)^{2}\left(-\phi_{y}+\frac{\partial w}{\partial y}\right)\right] \\
w(x, y, z, t)=w(x, y, t)
\end{gathered}
$$

where $u, v, w$ denote the displacement components in the $(x, y, z)$ directions at time $t ; u_{0}, v_{0}$ denote displacement of the middle surface; $\phi_{x}$ and $\phi_{y}$ are the angles of rotation of the transverse normal around $x$ and $y$ axes, respectively; and $h$ is the thickness of the composite laminate. The above relations can easily be reduced to a classical thin shell formulation by setting $\alpha=1, \beta=0, \gamma=0$. In this work, a single-layer shear deformation formulation, related to the Mindlin-Reissner theory of plates, is obtained by setting $\alpha=0, \beta=1, \gamma=0$ :

$$
u=u_{0}+z \phi_{x} \text { and } v=v_{0}-z \phi_{y}
$$

with $\phi_{x}, \phi_{y}$ given as:

$$
\phi_{x}=-\frac{\partial w}{\partial x}+\gamma_{x z} \text { and } \phi_{y}=\frac{\partial w}{\partial y}-\gamma_{y z}
$$




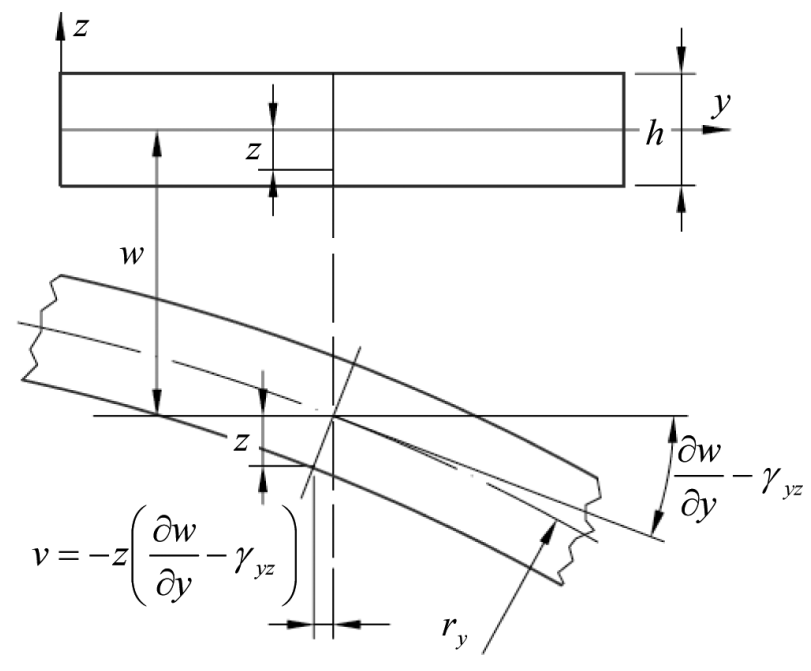

Fig. 1. Transverse shear deformations.

where $\gamma_{x z}, \gamma_{y z}$ are the transverse shear strains in the $x z$ and $y z$ planes, respectively.

Equations (2) and (3) express the following hypothesis: plane sections originally perpendicular to the middle surface remain plane after the deformation but not perpendicular to the middle surface (Fig. 1). The formulation is therefore related to the Mindlin-Reissner theory of plates.

The plate or shell considered in this work is assumed to be a laminated surface with thickness $h$. At any point of the shell, the membrane strains and curvatures in a rectangular coordinate system $(x, y, z)$ are given by the following equations:

$$
\begin{aligned}
& e_{x}=\frac{\partial u_{0}}{\partial x} \\
& e_{y}=\frac{\partial v_{0}}{\partial y} \\
& e_{x y}=\frac{1}{2}\left(\frac{\partial u_{0}}{\partial y}+\frac{\partial v_{0}}{\partial x}\right) \\
& \kappa_{x}=\frac{\partial \phi_{x}}{\partial x}=\frac{\partial}{\partial x}\left(-\frac{\partial w}{\partial x}+\gamma_{x z}\right) \\
& \kappa_{y}=\frac{\partial \phi_{y}}{\partial y}=\frac{\partial}{\partial y}\left(\frac{\partial w}{\partial y}-\gamma_{y z}\right) \\
& \kappa_{x y}=\frac{1}{2}\left(\frac{\partial \phi_{x}}{\partial y}+\frac{\partial \phi_{y}}{\partial x}\right)
\end{aligned}
$$

The transverse shear strains are calculated using the following equations:

$$
\begin{aligned}
\gamma_{x z} & =\frac{\partial w}{\partial x}+\phi_{x} \\
\gamma_{y z} & =\frac{\partial w}{\partial y}-\phi_{y}
\end{aligned}
$$

where $2 \varepsilon_{x z}=\gamma_{x z} ; 2 \varepsilon_{y z}=\gamma_{y z}$. 


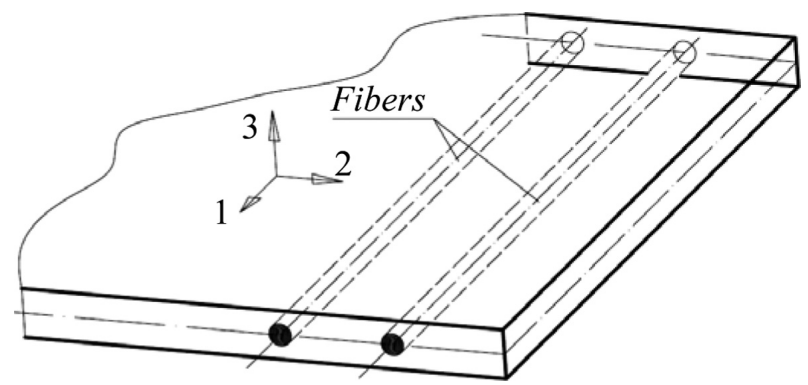

Fig. 2. Principal material directions for an individual layer; 1 is a direction of the fibers.

In Eqs (4)-(11) $z$ is a measure of the distance between the middle surface of the shell and the surface under consideration; $e_{x}, e_{y}$ are membrane strains; $e_{x y}$ is in-plane shear strain; and $\kappa_{x}, \kappa_{y}, \kappa_{x y}$ are curvatures at the midsurface in planes parallel to the $x z, y z$, and $x y$ planes, respectively; $u_{0}, v_{0}, w_{0}$ are the displacements of the middle surface along $x, y, z$ axes, respectively; $\gamma_{x z}, \gamma_{y z}$ are transverse shear strains in $x z$ and $y z$ planes; $\phi_{x}, \phi_{y}$ are angles of rotation of the cross-sections that were normal to the mid-surface of the undeformed shell (Fig. 1). The total normal strains due to both membrane and bending deformation in $x, y$ directions, respectively, can be written as follows:

$$
\varepsilon_{x}=e_{x}+z \kappa_{x} \text { and } \varepsilon_{y}=e_{y}-z \kappa_{y}
$$

\section{Lamina constitutive equations}

For a lamina of constant thickness $h$ and made of an orthotropic material (plate possesses a plane of elastic symmetry parallel to the $x y$ plane), the constitutive equations relating stresses $\sigma$ to strains $\varepsilon$ for a layer can be written in the principal material directions $(1,2,3)$ as:

$$
\left\{\begin{array}{l}
\sigma_{1} \\
\sigma_{2} \\
\sigma_{4} \\
\sigma_{5} \\
\sigma_{6}
\end{array}\right\}=\left[\begin{array}{ccccc}
Q_{11} & Q_{12} & 0 & 0 & 0 \\
Q_{12} & Q_{22} & 0 & 0 & 0 \\
0 & 0 & 2 Q_{44} & 0 & 0 \\
0 & 0 & 0 & 2 Q_{55} & 0 \\
0 & 0 & 0 & 0 & 2 Q_{66}
\end{array}\right]\left\{\begin{array}{c}
\varepsilon_{1} \\
\varepsilon_{2} \\
\varepsilon_{23} \\
\varepsilon_{13} \\
\varepsilon_{12}
\end{array}\right\}
$$

where the transverse normal stresses $\sigma_{3}$ are neglected. The principal material directions $(1,2,3)$ are shown in Fig. 2.

The quantities $Q_{i j}$ in Eq. (13) form the stiffness matrix and are expressed as follows [30,33]:

$$
\begin{aligned}
& Q_{11}=E_{11} / \Delta, \quad Q_{22}=E_{22} / \Delta, \quad Q_{12}=\nu_{21} E_{11} / \Delta \\
& Q_{44}=G_{23}, \quad Q_{55}=G_{13}, \quad Q_{66}=G_{12}, \quad \Delta=1-\nu_{12} \nu_{21}
\end{aligned}
$$

where $E_{11}, E_{22}$ are Young's moduli of the composite in 11 and 22 directions, respectively; $G_{23}, G_{13}, G_{12}$ are shear moduli of the composite in 23,13, and 12 directions, respectively; and $\nu_{12}$ is a Poisson's ratio in 12 direction (directions are defined as in the theory of elasticity, where the first subscript defines the normal to the plane under consideration and the second subscript defines the direction).

We note that $\varepsilon_{23}=\left(1 / 2 G_{23}\right) \sigma_{4}, \varepsilon_{13}=\left(1 / 2 G_{13}\right) \sigma_{5}$, and $\varepsilon_{12}=\left(1 / 2 G_{12}\right) \sigma_{6}$; thus, coefficients ' 2 ' appear in $Q_{i j}$ matrix.

The general transformation matrix from local to global coordinate system is given by matrix $\mathbf{T}$ :

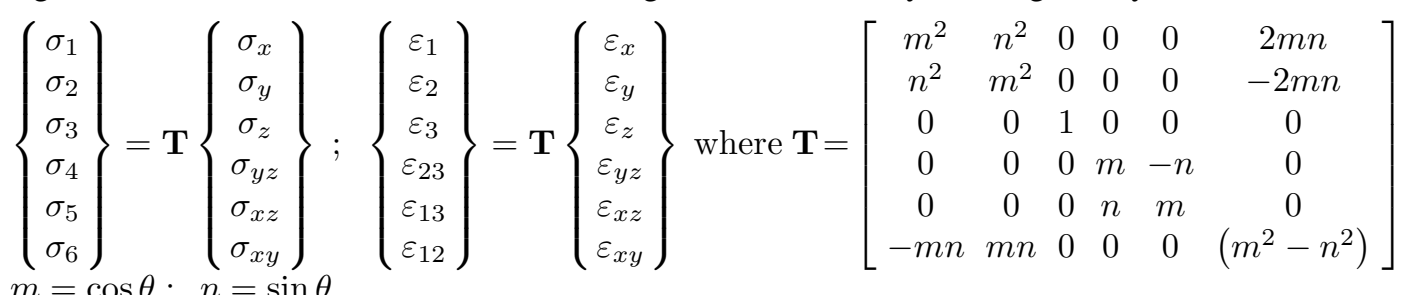




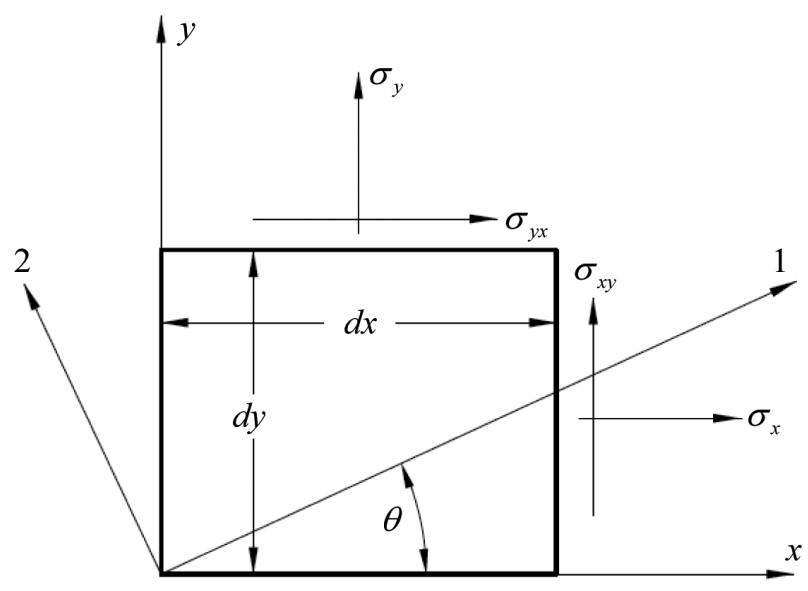

Fig. 3. Lamina coordinate system.

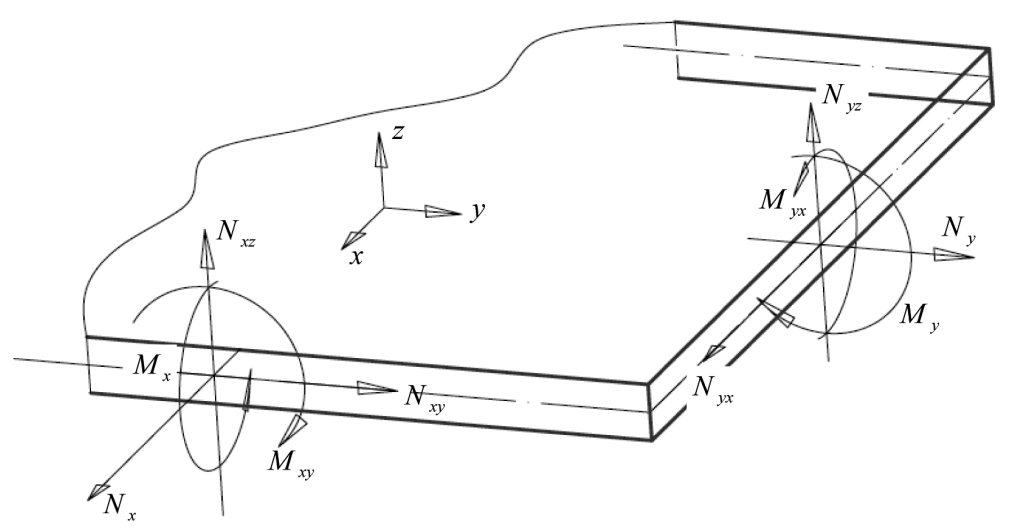

Fig. 4. Stress resultants on shell element.

where $\theta$ is the angle between a positive $x$ direction of a global coordinate system and a direction of the fibers 1 (Fig. 3). An inverse of the transformation matrix, $\mathbf{T}^{-1}$, can easily be found by replacing $\theta$ with $-\theta$ in $\mathbf{T}$.

Using Eqs (13)-(15) we can easily calculate the stresses in each lamina of the composite laminate. To calculate the stress resultants and stress couples $M_{x}, M_{y}, M_{x y}, N_{x}, N_{y}, N_{x y}, N_{x z}, N_{y z}$ in the laminate, we use definitions of normal and shear forces, as well as bending moments commonly used in beam, plate, or shell theory:

$$
\left\{\begin{array}{c}
N_{x} \\
N_{y} \\
N_{x y} \\
N_{x z} \\
N_{y z}
\end{array}\right\}=\int_{-h / 2}^{h / 2}\left\{\begin{array}{c}
\sigma_{x} \\
\sigma_{y} \\
\sigma_{x y} \\
\sigma_{x z} \\
\sigma_{y z}
\end{array}\right\} d z ; \quad\left\{\begin{array}{c}
M_{x} \\
M_{y} \\
M_{x y}
\end{array}\right\}=\int_{-h / 2}^{h / 2}\left\{\begin{array}{c}
\sigma_{x} \\
\sigma_{y} \\
\sigma_{x y}
\end{array}\right\} z d z
$$

The positive directions of the stress resultants, consistent with their definitions given by Eq. (16), are shown in Fig. 4. The shear correction factor is often applied in the definition of the transverse shear forces in Eq. (16). This is because transverse shear strains are defined according to the Mindlin plate theory, where the strain is constant over the thickness. The stresses are obtained by multiplication of strains by a shear modulus Eq. (13), which leads to constant distribution of the shear stresses across the thickness of the composite laminate. In isotropic shells, the shear stresses $\sigma_{x z}$ and $\sigma_{y z}$ are quadratic functions of thickness coordinate $z$, and in absence of the traction boundary conditions on the outer surfaces of the shell, they should vanish on the top and bottom surfaces, i.e., $z= \pm h / 2 \rightarrow \sigma_{x z}=\sigma_{y z}=0$. Determination of the shear correction factor is straightforward for isotropic shells but can be cumbersome for laminated shells with many layers, especially if they are dynamically loaded [19,38]. 


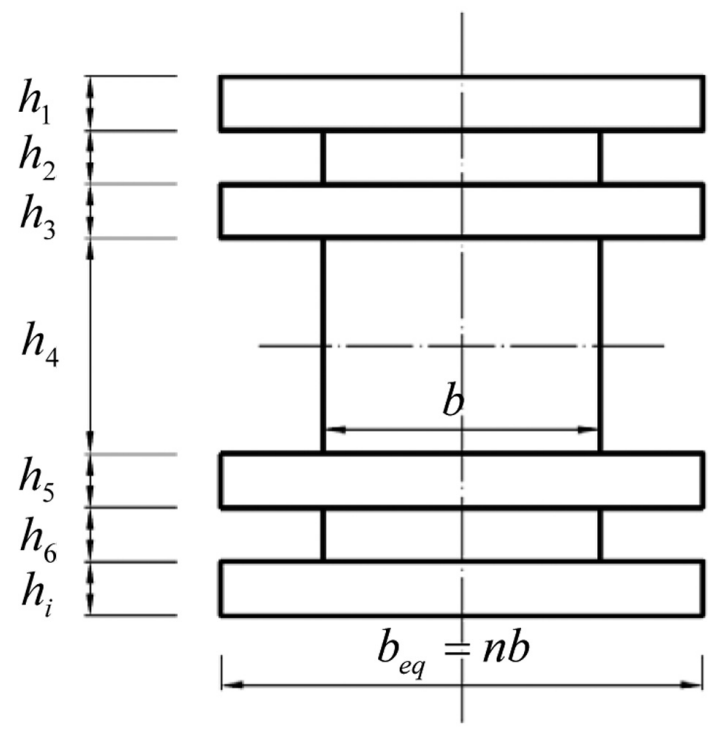

Fig. 5. Equivalent cross section of the composite beam.

In the current work we avoid computation of the shear correction factors altogether by using the transformed section method, which allows for accurate approximation of the distribution of the shear stresses through the thickness, without employing computationally expensive higher order kinematics of the shell. This approach has been used in the analytical calculation of composite beams, and it can easily be extended to plates and shells. In the case of the beam, the cross section built of several materials is transformed into an equivalent cross section of one material on which the resisting forces and the neutral axis are the same as on the original section. The usual flexure formula is then applied to the new section [31]. The equivalent cross section is found by multiplying the width of each lamina by a so-called modular ratio, given by:

$$
n=\frac{E_{x}}{E_{2}}
$$

where $E_{x}$ is Young's modulus of a considered lamina in the global $x$ direction and $E_{2}$ is Young's modulus of a lamina in the direction perpendicular to the fiber. We therefore have for the lamina oriented at $0^{\circ}, n=E_{1} / E_{2} \geqslant 1$ and for the lamina oriented at $90^{\circ}, n=1$. The width of each lamina is then multiplied by its respective modular ratio to form the equivalent cross section, shown in Fig. 5.

Having established the equivalent cross section, we can determine the equivalent area, first and second moment of area, which can be used to calculate the displacements, strains, and stresses. In the case of plates and shells, the geometric cross-section characteristics of individual laminas are multiplied by the modular factor given by:

$$
\begin{aligned}
& E_{e q u_{-} x}=E_{1} \cos ^{2} \theta+E_{2} \sin ^{2} \theta \\
& E_{e q u_{-} y}=E_{2} \cos ^{2} \theta+E_{1} \sin ^{2} \theta
\end{aligned}
$$

where $E_{e q u_{-} x}$ and $E_{e q u_{-} y}$ are the modular factors of considered lamina in global $x$ and $y$ directions, respectively; $E_{1}$ and $E_{2}$ are Young's moduli of considered lamina in directions 1 (fiber direction) and 2 (direction perpendicular to the fibers), respectively; and $\theta$ is the angle between a positive $x$ direction of a global coordinate system and a direction of the fibers 1, Fig. 3. The equivalent cross-section area of the considered lamina is therefore:

$$
A_{x i}=h_{i} E_{\text {equ_xi }} \text { where } i=1, k
$$

where $k$ is the number of laminas and $h_{i}$ is the thickness of the $i-t h$ lamina. The equivalent area of the laminate can be obtained by summation of equivalent areas:

$$
A_{x}=\sum_{i=1}^{k} A_{x i}
$$


Similarly, the equivalent first and second moment of area are given by:

$$
\begin{aligned}
Q_{x} & =\sum_{i=1}^{k} h_{i} z_{i} E_{\text {equ_xi }} \\
I_{x} & =\sum_{i=1}^{k}\left(\frac{h_{i}^{3}}{12}+h_{i} z_{i}^{2}\right) E_{\text {equ_xi }} \quad \text { where } i=1, k
\end{aligned}
$$

where $z_{i}$ is the distance between the center axes of the lamina and composite laminate.

The transverse shear stresses can now be calculated:

$$
\sigma_{x z}=\frac{Q_{x} N_{x z}^{\prime}}{I_{x}} ; \quad \sigma_{y z}=\frac{Q_{y} N_{y z}^{\prime}}{I_{y}}
$$

where $N_{x z}^{\prime}$ and $N_{y z}^{\prime}$ are transverse shear forces calculated not by means of Eq. (16), but through direct integration of transverse shear strains:

$$
N_{x z}^{\prime}=\int_{-h / 2}^{h / 2} G_{x z} \varepsilon_{x z} ; \quad N_{y z}^{\prime}=\int_{-h / 2}^{h / 2} G_{y z} \varepsilon_{y z}
$$

where $G_{x z}=G_{13}$ and $G_{y z}=G_{23}$. As mentioned above, application of the shear correction factor results in accurate computation of transverse shear forces. Substituting these forces into Eq. (22), along with previously obtained equivalent cross-section characteristics, allows for accurate determination of the non-continuous distribution of the transverse shear stresses across the thickness of the shell.

The above constitutive equations are universal for both plates and shells. They are implemented into the explicit finite element code EPSA. The composite shell element used is flat, with constant strains and 4 nodes and 5 degrees of freedom per node, i.e., linear velocities $\dot{u}, \dot{v}, \dot{w}$ in $x, y, z$ directions, respectively, and angular velocities $\dot{\phi}_{x}, \dot{\phi}_{y}$ around $y$ and $x$ axes, respectively. The curvature of the shell is modeled through finite element discretization. The positive directions of the degrees of freedom are the same as the positive directions of the stress resultants (Fig. 4).

Details of the kinematics of the element, integration scheme, and anti-hourglassing procedure are given in references [3,5].

\section{Rate-independent yield function for layered isotropic shells}

The yield surface discussed here is applied only to isotropic shells, analyzed by means of the layered method. This section is therefore not relevant for composite laminates.

As discussed in the Introduction, we use the von Mises yield criterion with elastic-perfectly plastic material representation in the analysis of layered isotropic shells. The structure under consideration is divided into layers across the thickness, and at every layer the yield condition is checked. This method, often called a layered method, requires integration of stresses through the thickness. It is therefore more expensive computationally than a stress resultant-based shell model used for non-layered isotropic shells in EPSA. The through-the-thickness-integration method is used in EPSA as a subsidiary of a composite shell model, hence the division into layers. It is often used for verification of the direct shell resultant-based shell model in EPSA [3,5].

We use in this work a von Mises yield criterion with transverse shear stresses accounted for, given by:

$$
F=\frac{1}{\sigma_{0}^{2}}\left[\sigma_{x}^{2}+\sigma_{y}^{2}-\sigma_{x} \sigma_{y}+3\left(\sigma_{x y}^{2}+\sigma_{x z}^{2}+\sigma_{y z}^{2}\right)\right]
$$

Because the elastic perfectly plastic material model is applied, no hardening function is defined. We assume additive decomposition of strains into elastic and plastic components:

$$
\varepsilon=\varepsilon^{e}+\varepsilon^{p}
$$

An associated flow rule is applied to determine incremental plastic strain components: 


$$
d \varepsilon^{p}=\lambda \frac{\partial F}{\partial \mathbf{S}}
$$

where the strains and stresses are represented by $5 \times 1$ matrices, given by:

$$
\begin{aligned}
\varepsilon & =\left\{\varepsilon_{x}, \varepsilon_{y}, \varepsilon_{x y}, \varepsilon_{x z}, \varepsilon_{y z}\right\} \\
\mathbf{S} & =\left\{\sigma_{x}, \sigma_{y}, \sigma_{x y}, \sigma_{x z}, \sigma_{y z}\right\}
\end{aligned}
$$

The following elastic law is applied:

$$
\mathbf{S}=\mathbf{E}\left(\varepsilon-\varepsilon^{p}\right)
$$

where $\mathbf{E}$ is the elastic shell stiffness matrix defined by Eqs (13)-(15) with angle $\theta=0$ for every layer.

The consistency condition ensures that the stresses remain on the yield surface:

$$
d F=\left(\frac{\partial F}{\partial \sigma_{x}}\right)^{T} d \sigma_{x}+\left(\frac{\partial F}{\partial \sigma_{y}}\right)^{T} d \sigma_{y}+\left(\frac{\partial F}{\partial \sigma_{x y}}\right)^{T} d \sigma_{x y}+\left(\frac{\partial F}{\partial \sigma_{x z}}\right)^{T} d \sigma_{x z}+\left(\frac{\partial F}{\partial \sigma_{y z}}\right)^{T} d \sigma_{y z}=0
$$

Or, more concisely:

$$
d F=\left(\frac{\partial F}{\partial \mathbf{S}}\right)^{T} d \mathbf{S}=0
$$

Substituting Eq. (26) into Eq. (28) and the resulting expression into Eq. (29) and solving for $\lambda$, we obtain:

$$
\lambda=\frac{\left(\frac{\partial F}{\partial \mathbf{S}}\right)^{T} \mathbf{E} d \varepsilon}{\left(\frac{\partial F}{\partial \mathbf{S}}\right)^{T} \mathbf{E} \frac{\partial F}{\partial \mathbf{S}}}
$$

The above equation is used in Eq. (26) to calculate the increments of plastic strain. Perfectly plastic behavior requires yielding of material (increase of plastic strain) without application of additional stresses. In this case, elasto-plastic stiffness is very small (not actually zero, to avoid numerical instability).

\section{Numerical examples}

The reliability of our approach is verified through a series of discriminating examples. The problems are selected to demonstrate and challenge new features introduced into the finite element code EPSA, i.e., representation of transverse shear deformation and transverse shear stresses in the analysis of layered isotropic shells and layered composites.

The importance of shear deformation in the analysis of composite structures is widely known and has been discussed by many authors. The objective of this section is to verify that the description of the shear effects presented here is reliable and leads to improvement in the accuracy of analyses of composite structures.

\subsection{Laminated composite strip under three-point bending}

We consider a simply supported 7-layer symmetric beam with a central line load. This problem is a commonly used composite benchmark [13]. The stacking sequence and orientation of the fibers is as follows: 0/90/0/90/0/90/0, where the centre ply is four times as thick as the others. The geometry of the beam with the stacking sequence is shown in Fig. 6.

The length of the beam is $L=50 \mathrm{~mm}$, the line load $P=10 \mathrm{~N} / \mathrm{mm}$ is applied at point $E$, and material properties are as follows: $E_{1}=1.0 E 5 \mathrm{MPa}, E_{2}=5.0 E 3 \mathrm{MPa}, G_{12}=3.0 E 3 \mathrm{MPa}, G_{13}=G_{23}=2.0 E 3 \mathrm{MPa}$, and $\nu_{12}=0.4, \nu_{23}=0.3$.

To verify that the current formulation features reliable representation of the shear effects and is therefore applicable to the analysis of thick composite shells, we investigate vertical displacement under the load (point $E$ ), bending stresses at the same location, and transverse shear stresses at point $D$ (Fig. 6). All of these values can be calculated 

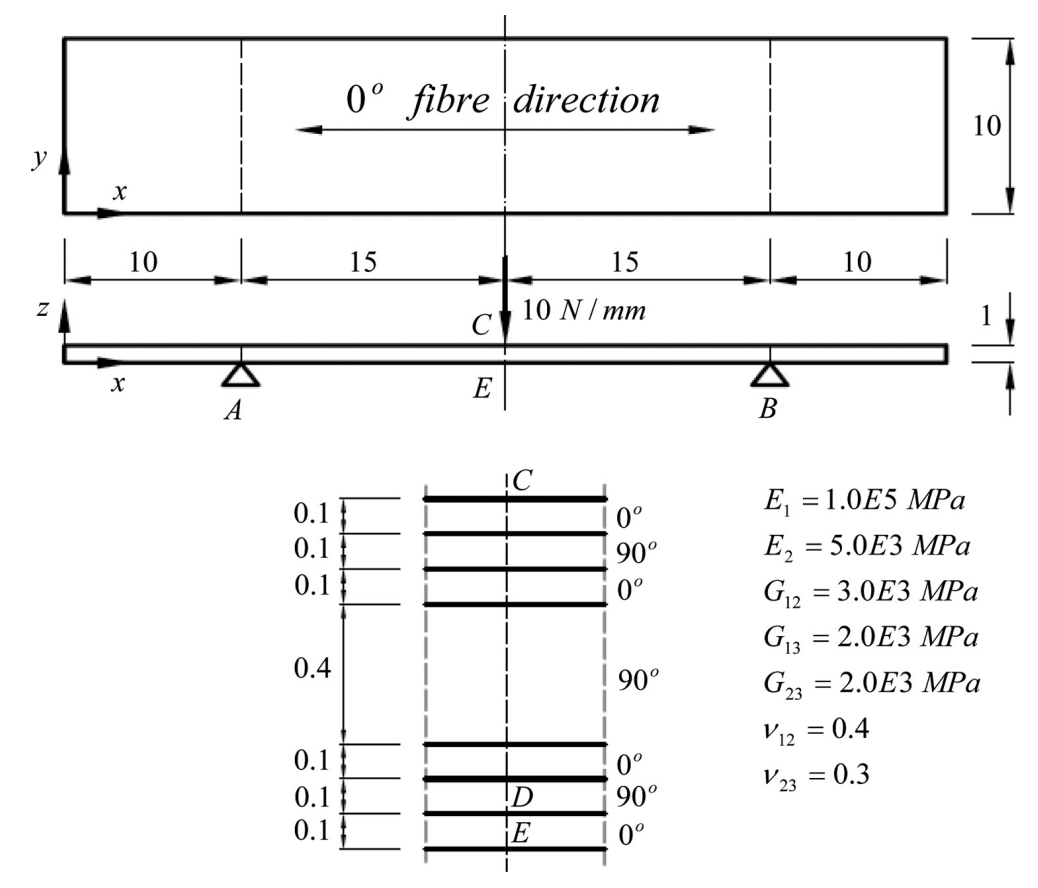

Fig. 6. Simply supported laminated beam subjected to central line load with material properties [13].

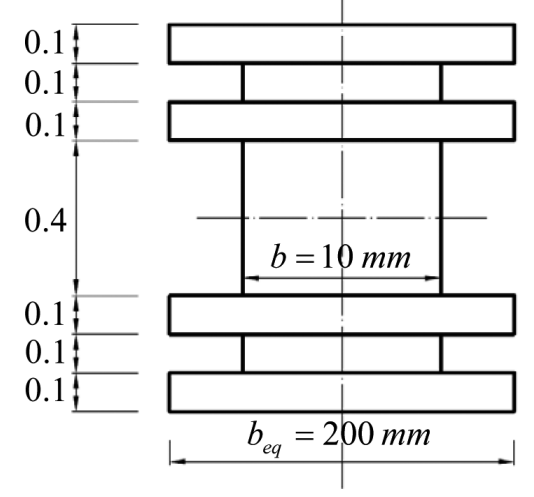

Fig. 7. Equivalent cross section of the composite beam.

analytically using a transformed section method. In this method, a cross section of several materials is transformed into an equivalent cross section of one material on which the resisting forces and neutral axis are the same as on the original section. The usual flexure formula is then applied to the new section [31].

The equivalent cross section is obtained by multiplying the width of every lamina by a so-called modular ratio, given by:

$$
n=\frac{E_{x}}{E_{2}}
$$

where $E_{x}$ is Young's modulus of a considered lamina in the global $x$ direction and $E_{2}$ is Young's modulus of the lamina in the direction perpendicular to the fiber. We therefore have for the lamina oriented at $0^{\circ}, n=E_{1} / E_{2}=20$, and for the lamina oriented at $90^{\circ}, n=1$. The width of every lamina is then multiplied by the appropriate modular ratio to form the equivalent cross section, shown in Fig. 7. 


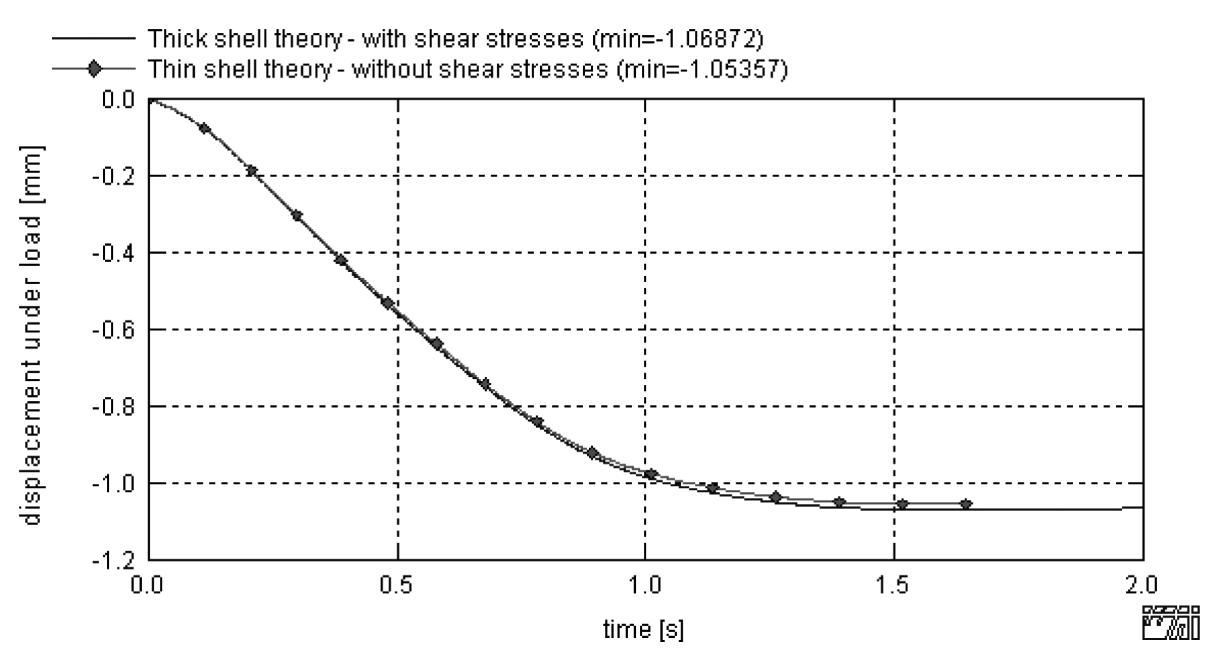

Fig. 8. Displacement time histories (displacement under load) with reduced .

Having established the equivalent cross section, we can determine its moment of inertia using Steiner's theorem (parallel axis theorem):

$$
I_{e q}=\sum_{j=1}^{m}\left(\frac{b_{j} h^{3}}{12}+b_{j} h d_{j}^{2}\right)_{j}=10.9667 \mathrm{~mm}^{4}
$$

where $m$ is a number of sub-areas Fig. 7; $b_{j}$ is a width of considered sub-area; $h$ is the total thickness of the composite laminate; and $d_{j}$ is the distance between the neutral axes of the laminate and lamina. Using the equivalent moment of inertia, we can calculate the displacement under load due to bending and shear actions. We apply the virtual unit force at point $C$, to determine the displacement by means of the virtual work principle.

$$
w=w_{M}+w_{Q}=\int_{L} \frac{M \bar{M}}{E_{2} I_{e q}} d x+\int_{L} \frac{6 Q \bar{Q}}{5 G_{13} A_{e q}} d x=\frac{P L^{3}}{48 E_{2} I_{e q}}+\frac{3 P L}{10 G_{13} A}=1.07 \mathrm{~mm}
$$

where $M, Q$ are bending moment and shear force functions, respectively; $\bar{M}, \bar{Q}$ are virtual bending moment and shear force, respectively; $L=30 \mathrm{~mm}$, (Fig. 6); $A$ is the area of the cross section.

The problem described above is modeled using 20 composite shell elements based on the thin shell and thick shell formulations. We compare the time histories obtained using the two formulations, as well as a final numerical solution to the analytical result given by Eq. (34). Figure 8 presents the time history for the vertical displacement at point $E$ (Fig. 6) calculated without accounting for shear stresses and after transverse shears have been introduced.

In Fig. 8, as expected, the displacement calculated with shear stresses taken into account $(w=-1.069 \mathrm{~mm})$ is slightly larger than that calculated with shear effects not taken into account $(w=-1.054 \mathrm{~mm})$. Both values, however, compare well with the reference solution $(w=-1.07 \mathrm{~mm})$. We therefore conclude that shear stresses do not significantly affect the response of the structure under consideration. The situation changes, however, if the transverse shear moduli $G_{13}$ and $G_{23}$ are significantly smaller than the in-plane shear modulus $G_{12}$. If $G_{13}=G_{23}=250 \mathrm{MPa}$, the displacement calculated using Eq. (34) is:

$$
\text { For } G_{13}=G_{23}=250 \mathrm{MPa}: \quad w=w_{M}+w_{Q}=-1.026-0.36=-1.386 \mathrm{~mm}
$$

The significant increase (nearly 26\%) of the displacement is caused by transverse shear action only, while the deformation caused by bending does not change. In this case, only a formulation that features a reliable representation of the shear effects is capable of delivering an accurate solution.

Figure 9 presents the time histories of displacement under load calculated, as previously, with EPSA composite shell elements based on the thin shell formulation, without taking shear stresses into account, and on the thick shell formulation, taking shear stresses into account. The significant difference between the results obtained by means of the thin and thick shell formulations is attributed to the influence of shear stresses. The current thick shell 
Table 1

Comparison of analytical and numerical results ( $A$ and $E$ are shown in Fig. 6)

\begin{tabular}{lcccc}
\hline & $\begin{array}{c}\text { Analytical } \\
\text { solution }\end{array}$ & $\begin{array}{c}\text { EPSA } \\
\text { thin shell }\end{array}$ & $\begin{array}{c}\text { EPSA thick } \\
\text { quadratic }\end{array}$ & $\begin{array}{c}\text { EPSA thick } \\
\text { TSM* }\end{array}$ \\
\hline Displacement at $E[\mathrm{~mm}]$ & 1.07 & 1.054 & 1.069 & 1.069 \\
Displacement at $E[\mathrm{~mm}]$, (reduced $\left.G_{13}\right)$ & 1.386 & 1.054 & 1.384 & 1.384 \\
Shear force between $A$ and $E[\mathrm{kN}]$ & 0.05 & 0.05 & 0.05 & 0.05 \\
Shear stress at $D(z= \pm 0.4 m m)[\mathrm{MPa}]$ & -4.103 & - & -2.620 & -4.131 \\
Bending moment at $E[\mathrm{kNm}]$ & $7.5 \mathrm{E}-4$ & $7.51 \mathrm{E}-4$ & $7.51 \mathrm{E}-4$ & $7.51 \mathrm{E}-4$ \\
Bending stress at $E[\mathrm{MPa}]$ & 683.89 & 684.96 & 684.96 & 684.96 \\
\hline
\end{tabular}

*Note: TSM - Transformed Section Method.

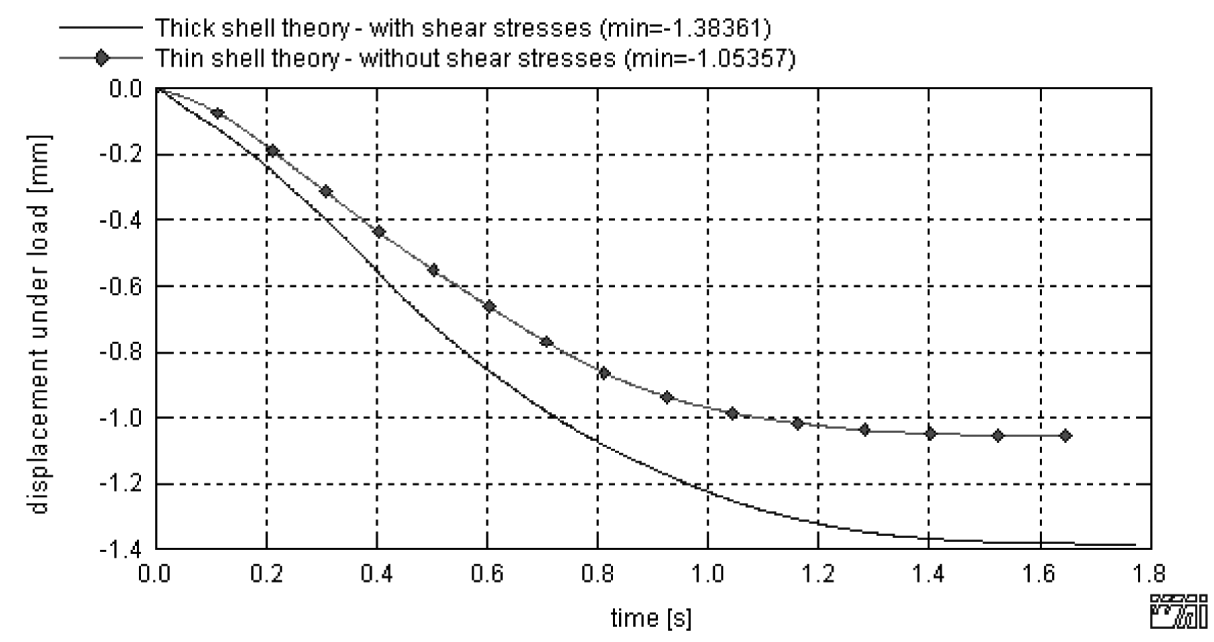

Fig. 9. Displacement time histories (displacement under load).

formulation delivers a value of displacement under load $(w=-1.3838 \mathrm{~mm})$ that practically matches the exact solution obtained by Eq. (35) $(w=-1.386 \mathrm{~mm})$. The thin shell formulation correctly predicts only the bending part of the deformation, while the shear part is neglected entirely Fig. 9.

For further verification, we compare the values of maximum bending stresses and transverse shear stresses computed using EPSA-III, with the reference solution calculated analytically. The maximum bending stress is given by:

$$
\sigma_{x}=\frac{n M}{I_{e q}} \frac{h}{2}= \pm \frac{n P L h}{8 I_{e q}}= \pm 683.89 M P a
$$

where $n$ is given by Eq. (32), $M$ is a bending moment at midspan of the beam (under the load), and $h$ is the thickness of the composite laminate. The transverse shear stress (interlaminar shear) between the $j$ th and the $(j+1) t h$ can be calculated from equilibrium of stresses. At the top layer $(z= \pm 0.4 \mathrm{~mm})$, we have:

$$
\sigma_{x z}=-\int_{0.4}^{0.5} \frac{\partial \sigma_{x}}{\partial x} d z=-\int_{0.4}^{0.5} \frac{\partial}{\partial x}\left(\frac{n 0.05 x z}{I_{e q}}\right) d z=-4.103 \mathrm{MPa}
$$

Table 1 compares the analytical and numerical values of displacements and bending stresses, as well as shear forces and bending moments, and Fig. 10 presents the transverse shear stress distribution.

The interlaminar shear stress at $z= \pm 0.4 \mathrm{~mm}$, determined using the current model, is $\sigma_{x z}=-4.134 \mathrm{MPa}$ (Fig. 10), which compares well with the analytical solution obtained by Eq. (37) $\left(\sigma_{x z}=-4.103 M P a\right)$. The distribution of the transverse shear stresses, as calculated by the transformed section method, also agrees closely with the reference solution. Table 1 and Fig. 10 compare the formulations. We note that, although application of the second-order shape function significantly improves model accuracy over the constant distribution, it nonetheless 
Table 2

Material properties and dimensions of the pinched cylinder

\begin{tabular}{ccc}
\hline \multicolumn{2}{c}{ Dimensions [mm] } & $L=100.0, r_{1}=27.0, r_{2}=25.0, r_{3}=23.0$ \\
\hline \multicolumn{2}{c}{ Loading } & Internal pressure: $p=100 \mathrm{MPa}$ \\
Material Properties & Layer 1 (Inner) & Isotropic: $E=2.1 E 5 \mathrm{MPa}, \nu=0.3$ \\
& Layer 2 (Outer) & Orthotropic: $E_{1}=1.3 E 5 \mathrm{MPa}, E_{2}=5.0 E 3 \mathrm{MPa}$, \\
& $\nu_{12}=0.3, G_{12}=1.0 E 4 \mathrm{MPa}, \mathrm{G}_{13}=500 \mathrm{MPa}$ \\
& NOTE: Direction 1 is a hoop direction; 2 is a $z$ direction \\
\hline
\end{tabular}

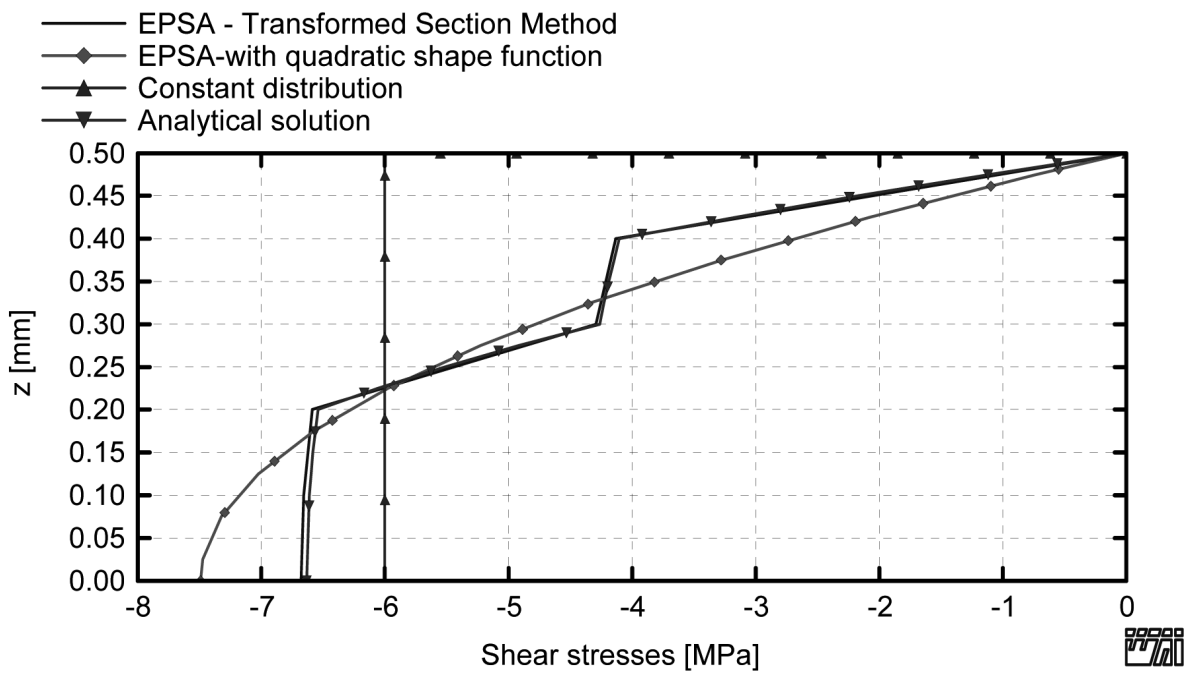

Fig. 10. Distribution of transverse shear stress $\sigma_{x z}[\mathrm{MPa}]$ across the thickness of the composite laminate (Axis of symmetry $z=0.0$ ).

leads to inaccuracies in shear stresses. This negatively affects the failure prediction capability of the model. The transformed section method allows for reliable shear stress distribution calculations, and it is simple and efficient. In all of the approaches considered, the integration of stresses through the thickness yields accurate result for shear forces.

The displacements calculated by means of the thin shell formulation are accurate only when shear effects are negligible. When the transverse shear modulus is significantly smaller than the in-plane shear modulus, the thin shell formulation does not produce accurate results. The displacements calculated by means of the thick shell formulation almost exactly match the reference solutions, verifying that the current thick shell formulation features reliable representation of shear effects and can be used successfully to model the behavior of thick composite plates, shells, and beams.

\subsection{Composite cylinder under internal pressure}

In the previous example, we verified that the current thick shell formulation correctly represents the increased shear flexibility caused by the differences between in-plane and out-of-plane shear moduli. Taking into account shear stresses in the analysis of thin shells may lead, however, to numerical deficiency commonly referred to as shear locking. A reliable shell model correctly predicts shear modes of deformation in the case of both thin and thick shells, without suffering from shear locking. To verify that this numerical deficiency is avoided here, we consider a long cylinder composed of two layers. The internal layer is an isotropic material and the external layer is an orthotropic material. The cylinder is subjected to internal pressure Fig. 11. Properties of the cylinder are given in Table 2.

Full symmetry is assumed and only an octant of the cylinder is considered. The mesh of 160 EPSA-III shell elements, used to model an octant of the cylinder, is shown in Fig. 12. We investigate radial displacement of the cylinder Fig. 11, calculated by means of the thin and thick shell formulations. The outer shell layer is orthotropic, 


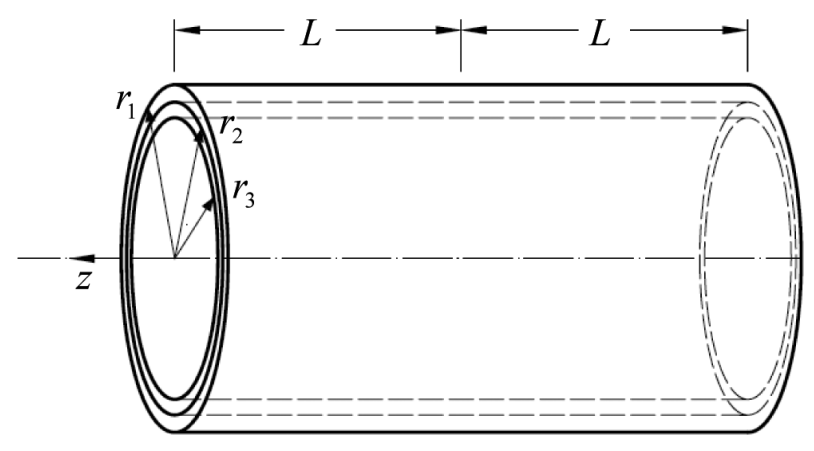

Fig. 11. Composite cylinder under internal pressure.

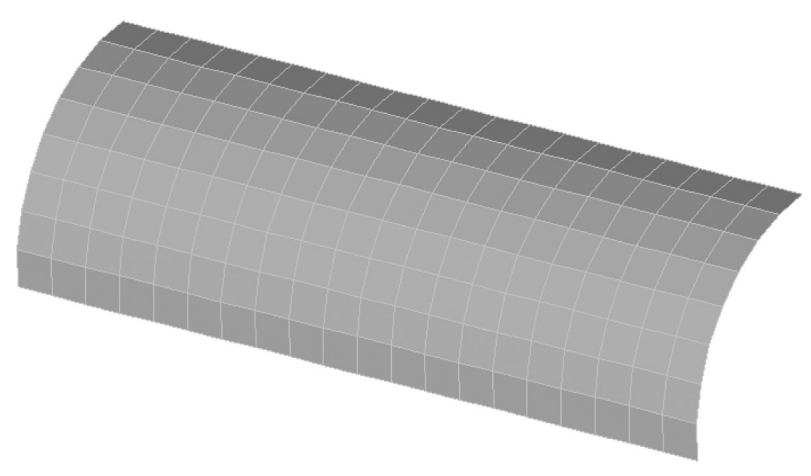

Fig. 12. Finite element mesh of an octant of the cylinder.

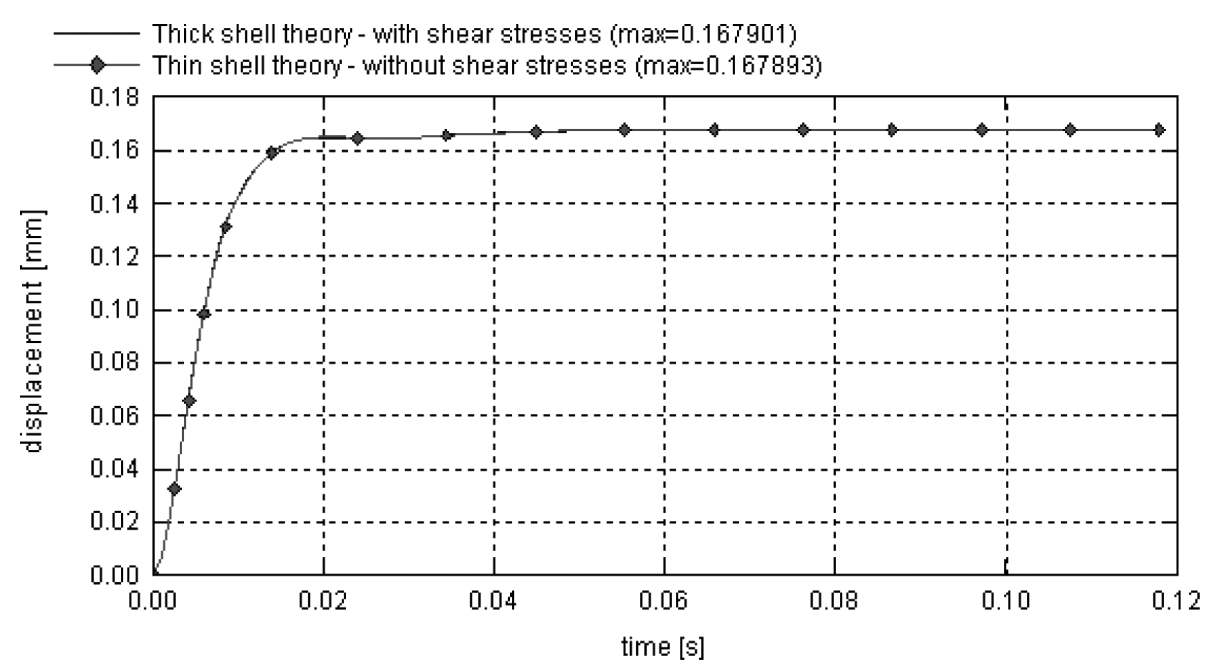

Fig. 13. Time history plots for radial displacements.

the inner layer is isotropic, and the cylinder is relatively thin. The loads are resisted mainly by the membrane action of the shell, and shear effects are negligible. Despite the fact that the in-plane shear modulus $\left(G_{12}\right)$ of layer 2 is much larger than the out-of-plane shear modulus $\left(G_{13}\right)$, the difference between displacements obtained from the two formulations should not be significant. The time histories of displacement under load determined using the thin and thick shell formulations are shown in Fig. 13.

We see in Fig. 13 that the radial displacements calculated using the thin and thick shell formulations practically 


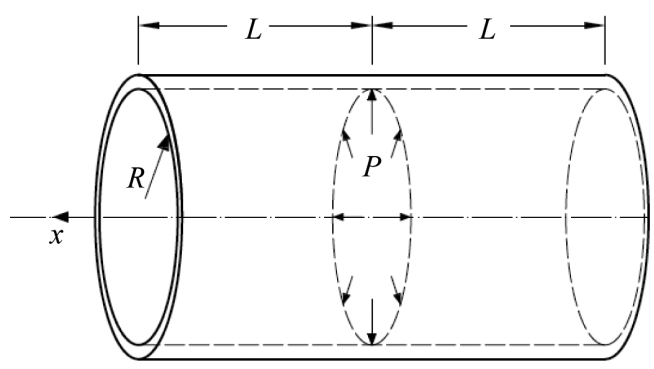

$$
\begin{aligned}
& L=100 \mathrm{~mm} \\
& R=25 \mathrm{~mm} \\
& t=0.5 \mathrm{~mm} \\
& E=2.1 E 5 \mathrm{MPa} \\
& v=0.3 \\
& \sigma_{o}=215 \mathrm{MPa} \\
& P=0.0625 \mathrm{kN} / \mathrm{mm}
\end{aligned}
$$

Fig. 14. Cylindrical shell subjected to a ring of pressure.

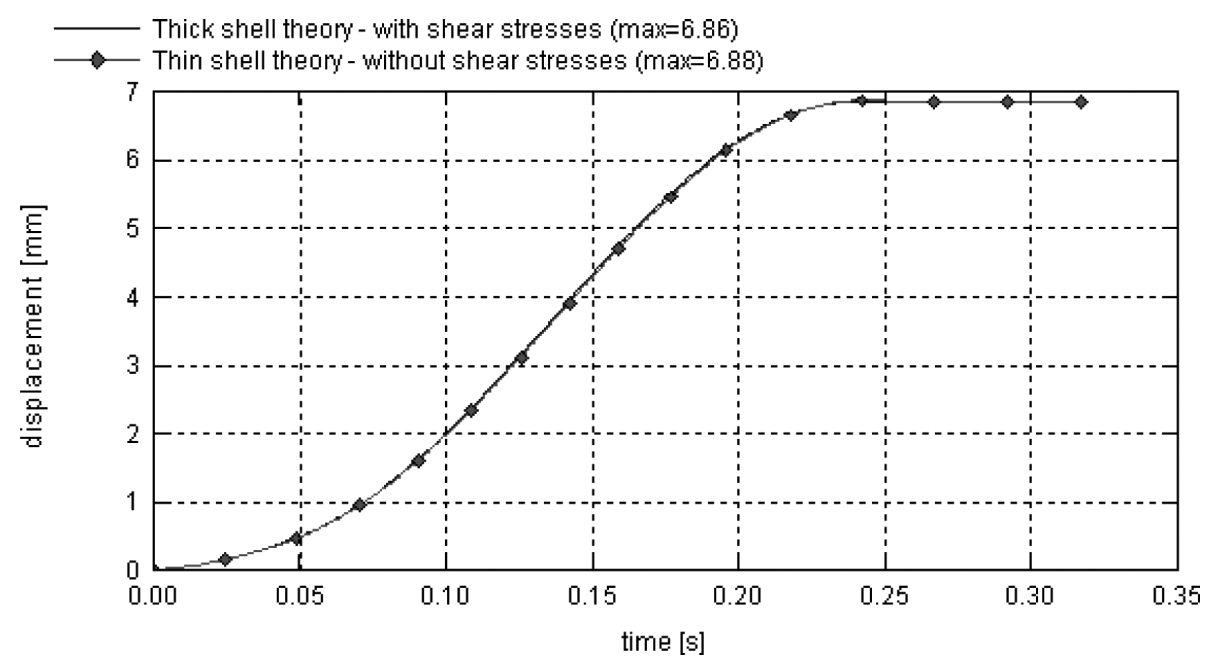

Fig. 15. Radial displacements for a cylinder subjected to a ring of pressure $(t=0.5 \mathrm{~mm})$.

coincide. Because, as discussed above, shear effects are negligible, this result proves the reliability of the thick shell formulation.

\subsection{Cylindrical shell subjected to a ring of pressure}

As discussed in the Introduction, the main objective of this work is to account for the influence of transverse shear strains and stresses on the behavior of composite laminates and elasto-plastic isotropic shells in analyses using a layered method (through-the-thickness integration). In Sections 6.1 and 6.2, the composite problems were investigated, verifying the reliability of the current thick shell formulation for composite structures. In this section, we verify the dependability of the representation of the transverse shear stresses in the layered isotropic shells model in EPSA.

We consider a cylindrical shell under the ring of pressure. The geometry and material parameters are shown in Fig. 14. Due to symmetry, we need consider only an octant of a shell, which is modeled using finite element mesh, as shown in Fig. 12.

We analyze the problem using EPSA layered shell elements, based on the thin shell formulation, and compare the results with those obtained using the same shell elements modified to account for transverse shear deformation. First, we consider a cylinder with thickness $t=0.5 \mathrm{~mm}$. Shells in which the ratio of the radius of the curvature and thickness is higher than 50 usually are considered thin. In this case, the results delivered by EPSA thin-layered shell element, previously shown to be reliable, will be sufficiently accurate. Solving the problem using the thick layered shell formulation presented here should therefore produce similar results. We compare the radial displacements at midspan of the cylinder, determined by thin and thick shell formulations. The time history plots are shown in Fig. 15. 
Thick shell theory - with shear stresses ( $\max =10.77367$ )

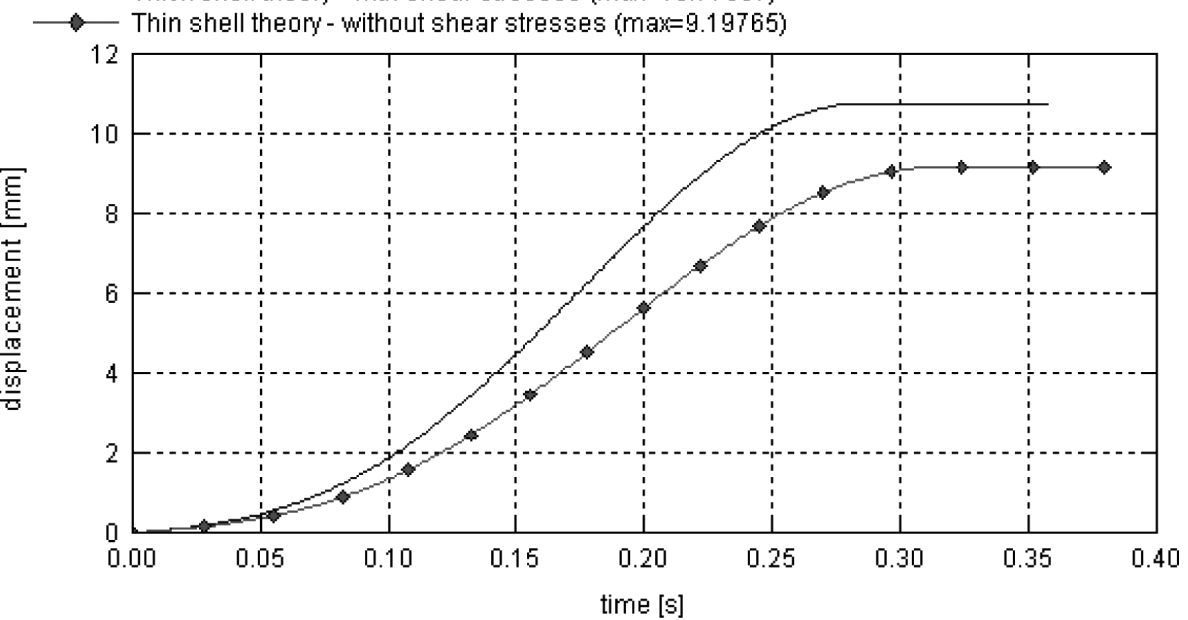

Fig. 16. Radial displacement for a cylinder subjected to a ring of pressure $(t=5 \mathrm{~mm})$.

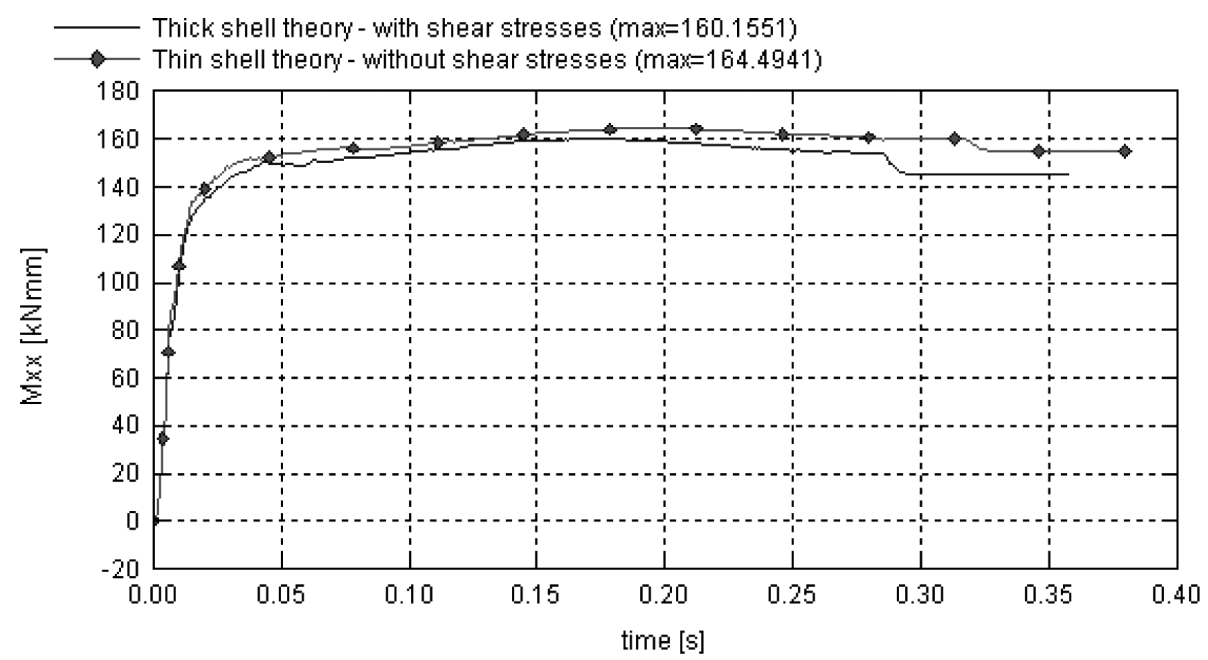

Fig. 17. Bending moment at midspan of the cylinder subjected to a ring of pressure $(t=5 \mathrm{~mm})$.

Figure 15 shows that, as expected, the results produced by the thin and thick shell formulations are practically identical. Shear effects are negligible in this problem, as correctly recognized by the thick layered shell model.

We increase the thickness of the cylinder to $t=5 \mathrm{~mm}$ and the pressure to $P=1.0 \mathrm{kN} / \mathrm{mm}$ and again compare the radial displacements obtained from the two formulations. The displacement time history plots are shown in Fig. 16.

Displacement calculated using the current, thick shell formulation is $17 \%$ larger than that obtained without accounting for shear stresses. At the same time, the bending moments and axial forces determined by the thick and thin shell formulations are approximately the same Fig. 17. This significant discrepancy between displacement values is attributed to increased influence of the transverse shear effects, which are correctly represented in the current model.

\section{Conclusion}

Accounting for out-of-plane shear strains and stresses is necessary to accurately model the elasto-plastic behavior of thick plates and shells. In the case of composite laminates, the influence of transverse shear stresses is even 
more important. This is due to the orthotropic nature of these structures and the often significant difference between in-plane and out-of-plane shear moduli. Because this leads to increased shear flexibility of the composites, neglecting shear effects may result in an unsafe design. Moreover, transverse shear stresses cause delamination in composites, one of the most important failure modes. A thick shell formulation makes it possible to account for transverse shear stresses in failure criteria. With the use of appropriate experimental data, this can result in greatly improved failure predictions for composite laminates.

EPSA is an explicit code that features a constant strain, quadrilateral shell element. The equations of motion are solved locally, without assembling the stiffness matrix of the structure [1-3,5]. Neither shear nor membrane locking is experienced, as shown in Section 7. The anti-hourglass procedure is given in reference [11].

The numerical examples in Section 7 were selected to challenge the most important features of this work, i.e., representation of transverse shear effects in elasto-plastic investigations of isotropic shells and composite laminates. In all of the analyzed cases, the results are accurate, verifying that the model is well grounded. EPSA layered shell and composite finite elements are therefore capable of delivering accurate approximations of the structural behavior of thin and thick plates, shells, and composite laminates for considered types of problems represented by the examples discussed in this paper.

\section{References}

[1] R.S. Atkatsh, M.L. Baron and M.P. Bieniek, A Finite Difference Variational Method for Bending of Plates, Computers \& Structures 11 (1980), 573-577.

[2] R.S. Atkatsh, M.P. Bieniek and M.L. Baron, Dynamic Elasto-Plastic Response of Shells in an Acoustic Medium - EPSA Code, Int J Num Meth Eng 19(6) (1983), 811-824.

[3] R.S. Atkatsh, M.P. Bieniek and I.S. Sandler, Theory of Viscoplastic Shells for Dynamic Response, J of Applied Mechanics 50 (1983), 131-136.

[4] Y. Basar, Y. Ding and R. Schultz, Refined shear-deformation models for composite laminates with finite rotations, Int J Solids Structures 30(19) (1993), 2611-2638.

[5] M.P. Bieniek and J.R. Funaro, Elasto-plastic behaviour of plates and shells, Technical report DNA 3584A, Weidlinger Associates, New York, 1976.

[6] W. Chen and D. Han, Plasticity for Structural Engineers, Springer-Verlag, 1988.

[7] R.D. Cook, Two hybrid elements for analysis of thick and thin sandwich plates, Int J Num Meths Eng 5 (1972), 277.

[8] G.A.O. Davis, Benchmarks For Composite Delamination, NAFEMS, Ref: R0084, Issue 1, 2002.

[9] S.T. Dennis and A.N. Palazotto, Transverse Shear Deformation in Orthotropic Cylindrical Pressure Vessels Using a Higher-Order Shear Theory, AIAA Journal 27(10) (1989), 1441-1447.

[10] D.C. Drucker, Limit Analysis of Cylindrical Shells Under Axially Symmetric Loading, Proc. 1st Midwestern Conf. Solid Mech., Urbana, Illinois, 1954, 158-163.

[11] D.P. Flanagan and T. Belytschko, A uniform strain hexahedron and quadrilateral with orthogonal hourglass control, Int J Num Meth Eng 17 (1981), 679-706

[12] W. Flugge, Stresses in Shells, Springer, New York, 1960.

[13] S. Hardy, Composite Benchmarks, NAFEMS, Ref: R0031, Issue 2, 2001.

[14] H.-C. Hu, Variational Principles of Theory of Elasticity with Applications, Scientific Publisher, Beijing, China, 1984.

[15] P.G. Hodge, Plastic Analysis of Structures, McGraw-Hill, 1959.

[16] A.A. Iliushin, Plastichnost', Gostekhizdat, Moscow, 1956.

[17] W.B. Kratzig, 'Best' transverse shearing and stretching shell theory for nonlinear finite element simulations, Comp Meth Appl Mech Eng 103 (1992), 135-160.

[18] W.B. Kratzig and D. Jun, On 'best' shell models-from classical shells, degenerated and multi-layered concepts to 3D, Archive of Applied Mechanics 73 (2003), 1-25.

[19] S.V. Kulkarni and N.J. Pagano, Dynamic Characteristics of Composite Laminates, J of Sound and Vibrations 23(1) (1972), $127-143$.

[20] A.K. Noor and W.S. Burton, Assessment of shear deformation theory for multilayered composite plates, Appl Mech Rev 42(1) (1989), $1-13$.

[21] C.B. Norris, Strength of Orthotropic Materials Subjected to Combined Stress. Forest Product Lab. Report 1816, $1950,1962$.

[22] A.N. Palazotto and P.E. Linnemann, Vibration and buckling characteristics of composite cylindrical panels incorporating the effects of a higher order shear theory, Int J Solids Structures 28(3) (1991), 341-361.

[23] B.N. Pandya and T. Kant, Higher order shear deformable theories for flexure of sandwich plates, Int J Solids Structures 24 (1988), 1267-1286.

[24] N.S. Putcha and J.N. Reddy, A refined mixed shear flexible finite element for the nonlinear analysis of laminated plates, Computers and Structures 22(4) (1986), 529-538.

[25] J.N. Reddy, A simple high-order theory for laminated composite plates, J Appl Mech 51 (1984), 745-752.

[26] J.N. Reddy, On refined computational models composite laminates, Int J Num Meths Eng 27 (1989), 361-382. 
[27] J.N. Reddy, E.J. Barbero and J.L. Teply, A plate bending element based on a generalized laminate plate theory, Int J Num Meths Eng 28 (1989), 2275-2292.

[28] E. Reissner, The effects of transverse shear deformation on the bending of elastic plates, J Appl Mech ASME 12 (1945), 66-77.

[29] E. Reissner, On transverse bending of plates, including the effects of transverse shear deformation, Int J Solids Structures 11 (1975), $569-576$.

[30] J.G. Sloan, The Behavior of Rectangular Composite Material Plates Under Lateral and Hydrothermal Loads, MMAE Thesis. University of Delaware, 1979.

[31] A.C. Ugural and S.K. Fenster, Advanced Strength and Applied Elasticity, Third Edition, Prentice Hall, 1995.

[32] A.R. Venson and G.Z. Voyiadjis, Damage quantification in metal matrix composites, J Engng Mech 127 (2001), 291-298.

[33] J.R. Vinson and R.L. Sierakowski, The Behavior of Structures Composed of Composite Materials, Kluwer Academic Publishers, 1987.

[34] G.Z. Voyiadjis and B. Deliktas, A coupled anisotropic damage model for the inelastic response of composite materials, Comp Meth Appl Mech Engn 183 (2000), 159-199.

[35] G.Z. Voyiadjis and T. Park, Local and interfacial damage analysis of metal matrix composites using finite element method, Engn Fracture Mech 56(4) (1997), 483-511.

[36] G.Z. Voyiadjis and A.R. Venson, Experimental damage investigation of a SiC-Ti aluminade metal matrix composite, Int J Damage Mech 4 (1995), 338-361.

[37] G.Z. Voyiadjis and P. Woelke, General non-linear finite element analysis of thick plates and shells, Int J Solids Structures $43(7-8,1)$ (2005), 2209-2242.

[38] J.M. Whitney, Shear Correction Factors for Orthotropic Laminates under Static Load, $J$ of Applied Mechanics, Transactions of ASME (1973), 302-304. 

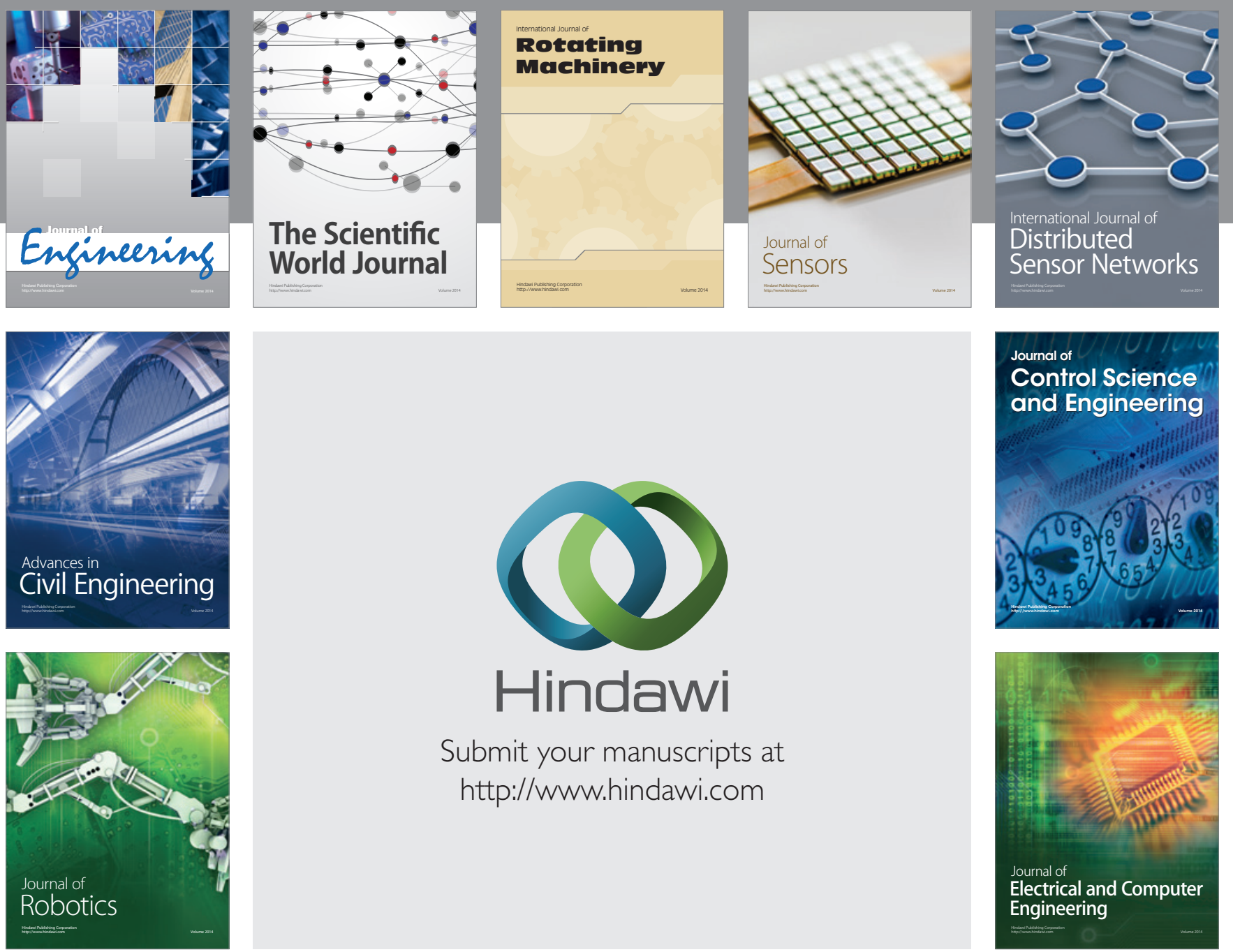

Submit your manuscripts at

http://www.hindawi.com
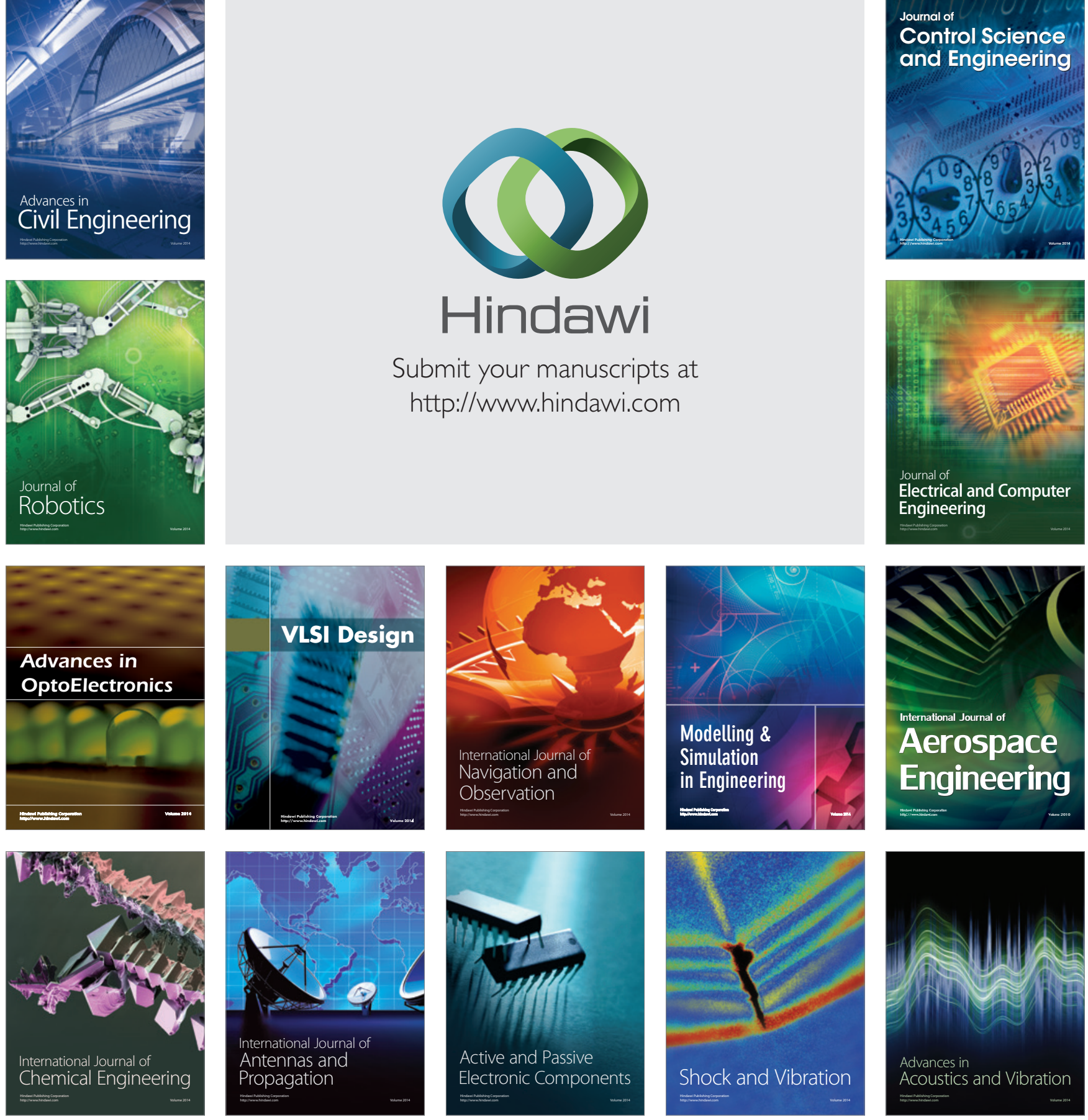